\title{
Persistent Hypoglycemia with Polycystic Kidneys: A Rare Combination - A Case Report
}

\author{
Priya Prasher $^{a} \quad K^{2}$ atherine Redmond ${ }^{a}$ Hillarey Stone ${ }^{b} \quad$ James Bailes $^{c}$ \\ Edward Nehus $^{b}$ Deborah Preston ${ }^{a}$ Joseph Werthammer ${ }^{a}$ \\ a Department of Pediatrics, Marshall University, Joan C Edwards School of Medicine, \\ Huntington, WV, USA; ${ }^{b}$ Division of Nephrology and Hypertension, Cincinnati Children's \\ Hospital Medical Center, Cincinnati, OH, USA; 'Division of Endocrinology, Hoops Family \\ Children's Hospital at Cabell Huntington Hospital, Huntington, WV, USA
}

\section{What Is It about?}

Our case report is about the first case of an infant with persistent hypoglycemia and congenital polycystic kidney disease reported in the USA. This unusual cooccurrence was linked to an autosomal recessive genetic variant that was previously described in 11 European families. Diamox was begun at 2 weeks of age, with no further episodes of hypoglycemia observed.

\section{Keywords}

Polycystic kidney disease $\cdot$ Hyperinsulinemic hypoglycemia $\cdot$ PMM2 gene mutation

\begin{abstract}
We present the case of an infant referred to our NICU born at 39 weeks' gestation with persistent hypoglycemia with elevated insulin levels $(\mathrm{HI})$ requiring diazoxide to maintain normoglycemia. Additionally, polycystic kidney disease (PKD) was detected by ultrasound. Molecular genetic testing revealed pathogenic variants in the PMM2 gene, i.e., a variant in the promoter region and a missense variant in the coding region. The precoding variant was recently described in 11 European families with similar phenotypes, either in a homozygous state or as compound heterozygous with a pathogenic coding variant. In neonates with $\mathrm{HI}$ associated with PKD, this rare recessive disorder should be considered.




\section{Established Facts}

- The genetic cooccurrence of hyperinsulinemic hypoglycemia and congenital polycystic kidney disease has only been described once in the nephrology literature, involving 17 children from 11 European families.

\section{Novel Insights}

- This report might be helpful in alerting neonatologists of this rare association and facilitating prompt diagnosis and treatment.

- This is the first case reported in the USA.

\section{Introduction}

Hyperinsulinemic hypoglycemia (HI) is a rare cause of persistent hypoglycemia in neonates and children and can lead to brain damage [1]. Congenital polycystic kidney disease (PKD) typically presents perinatally with enlarged echogenic kidneys with fusiform dilatation of the collecting ducts and varying degrees of congenital hepatic fibrosis [2]. The cooccurrence of these two rare disorders linked to PMM2 variants was reported in 11 European families by Cabezas et al. [3] in 2017. We present an additional case of this cooccurrence with the PMM2 variant. It confirms the first report and, to our knowledge, represents the first case from North America.

\section{Patient Information}

A 1-week-old female infant of European descent was referred to our NICU from an outlying hospital for concerns of persistent hypoglycemia despite multiple glucose boluses and parenteral glucose infusion. The patient was born at 39 weeks' gestational age to a 25 -year-old G1 mother by spontaneous vaginal delivery. Pregnancy was uncomplicated and negative for maternal diabetes. Mother's prenatal laboratory studies and ultrasounds were unremarkable. The patient's birth weight was 4,119 g ( $>90$ th percentile). Blood culture at the outlying facility was negative. Aside from the persistent hypoglycemia, the baby demonstrated no other apparent health issues at the referring hospital.

\section{Clinical Findings}

The glucose level upon admittance to our NICU was $31 \mathrm{mg} / \mathrm{dL}$, requiring a $10 \%$ dextrose in water (D10W) bolus at $2 \mathrm{~mL} / \mathrm{kg}$, followed by an intravenous glucose infusion rate of 10 $\mathrm{mg} / \mathrm{kg} / \mathrm{min}$. The rate was increased to $15 \mathrm{mg} / \mathrm{kg} / \mathrm{min}$ to maintain glucose levels above 40 $\mathrm{mg} / \mathrm{dL}$. The insulin level was $19.6 \mathrm{uIU} / \mathrm{mL}$ (normal range: 2.6-24.9) and the c-peptide level was $4.1 \mathrm{ng} / \mathrm{mL}$ (normal range: 1.1-4.4) during the initial episode of hypoglycemia. Insulin levels remained elevated during subsequent episodes of hypoglycemia.

On physical examination, bilateral renal enlargement was noted, and an abdominal ultrasound showed bilateral polycystic kidneys (Fig. 1). 
Biomedicine Hub

Fig. 1. Left kidney showing multiple cysts of various sizes.

\begin{tabular}{|c|c|}
\hline \multicolumn{2}{|l|}{ Biomed Hub 2020;5:511389 } \\
\hline DOI: 10.1159/000511389 & $\begin{array}{l}\text { (c) } 2020 \text { The Author(s). Published by S. Karger AG, Basel } \\
\text { www.karger.com/bmh }\end{array}$ \\
\hline
\end{tabular}
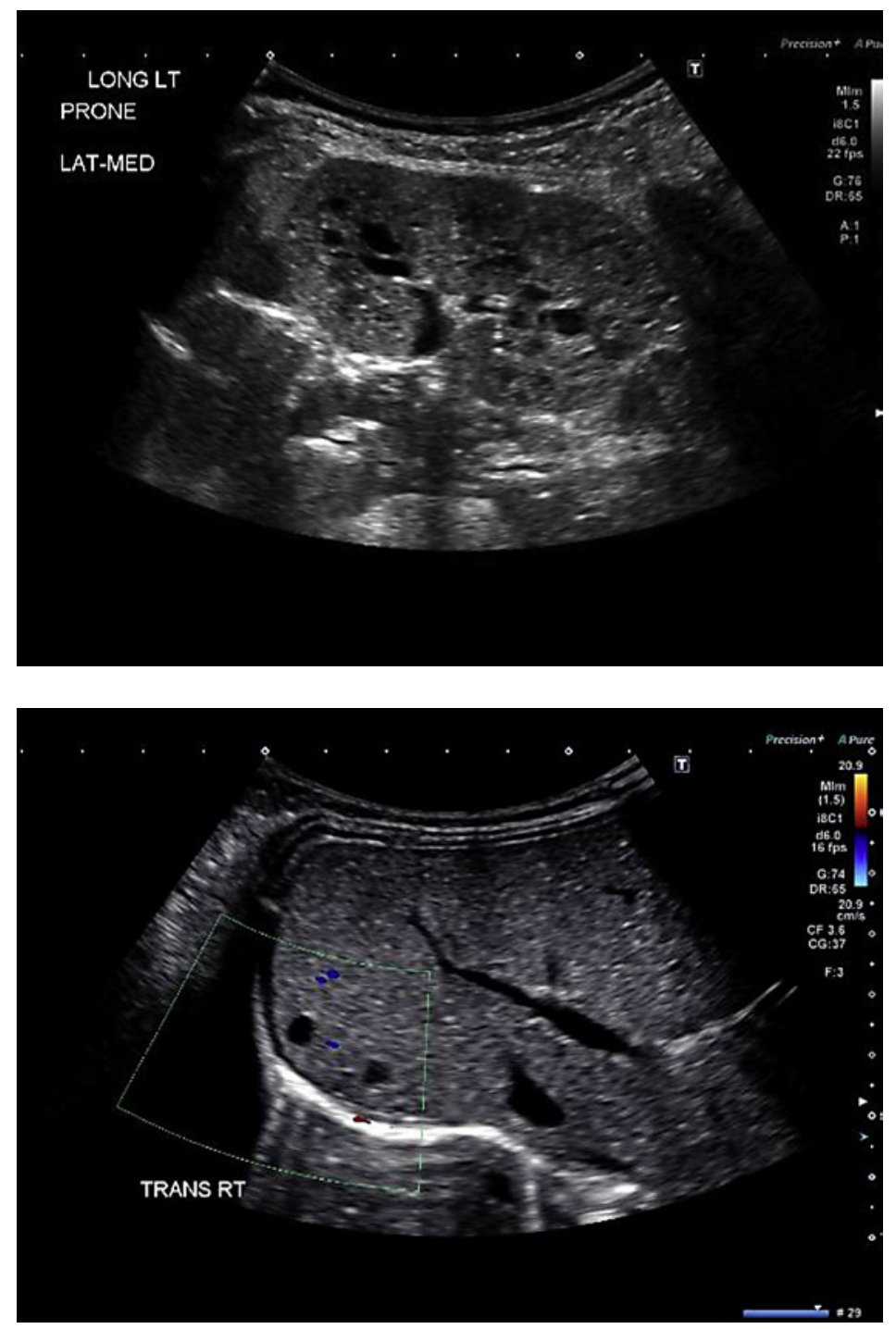

Fig. 2. Liver with multiple, scattered cysts.

\section{Therapeutic Intervention}

Diazoxide at $10 \mathrm{mg} / \mathrm{kg} / 24 \mathrm{~h}$ divided into 3 daily doses was begun at 2 weeks of age, and glucose infusion was weaned when bolus feeding with a $20 \mathrm{kcal} / \mathrm{oz}$ formula was initiated. The volume of feeding was increased gradually, while parenteral fluid was weaned. Preprandial point-of-care blood glucose levels remained $>50 \mathrm{mg} / \mathrm{dL}$. The patient was discharged at 3 weeks of life on diazoxide and formula feeds without dietary restrictions.

\section{Follow-Up}

Given the findings of $\mathrm{HI}$ and polycystic kidneys, the patient was followed by endocrinology, nephrology, and genetics. Given the clinical concern for HI and PKD (HIPKD), targeted molecular genetic testing was performed to evaluate for variants in the phosphomannomutase 2 (PMM2) gene (NM_000303.2). Other genes associated with ARPKD (PKHD1 and $D Z I P 1 L)$ were also analyzed. Targeted family testing was performed in both parents. The 
patient was compound heterozygous for a paternally inherited missense variant (c.422G>A, p.Arg141His) and a maternally inherited variant in the precoding region (c.-167G>T). Repeat abdominal ultrasound at 3 months of age revealed many renal cortical cysts bilaterally as well as scattered cysts in the liver (Fig. 2). Daily point-of-care glucose measurements have remained normal on an unrestricted diet and diazoxide, and no surgical treatment such as pancreatectomy has yet been considered.

\section{Diagnostic Findings}

HIPKD secondary to variants in the PMM2 gene was found.

\section{Discussion}

Both autosomal recessive PKD (ARPKD) and congenital HI are rare disorders. Patients with ARPKD often have the combination of polycystic kidneys with abnormal hepatic findings and oligohydramnios [4, 5], while HI patients typically have gene mutations resulting in abnormal regulation of insulin from their pancreas [6,7]. The cooccurrence of these two rare diseases have previously been described in patients with pathogenic variants in PMM2 [5].

The c. $-167 \mathrm{G}>\mathrm{T}$ variant in the promotor region of the PMM2 gene, either in the homozygous state or in a heterozygous conformation with a concurrent variant in the coding region, was recently reported to be causative of disease in 17 patients from 11 unrelated European families who presented with autosomal recessive HI and PKD [3]. All 17 patients were found to have cystic kidney disease, detected on antenatal imaging or incidentally in childhood. Two of the patients progressed to end-stage renal disease, requiring dialysis or transplantation by ages 2 and 20 years. All patients had coexisting HI that was diagnosed at a median age of 10 months, usually presenting with hypoglycemic seizures. The majority of those patients responded to treatment with diazoxide, while some did well without therapy. Many of the patients were found to have liver cysts on imaging, with little or no abnormalities in liver function tests. Liver involvement did not result in portal hypertension, a complication seen in many patients with ARPKD. Patients did not exhibit dysmorphic features or abnormalities in other organs. Of note, 6 patients in the previous report had the same variants identified in our patient (c.-167G>T/c.422G>A) [3]. No clear renal genotype-phenotype correlation is evident; 1 patient with these variants progressed to end-stage renal disease by age 2 years while others maintained normal kidney function for many years following diagnosis. Patients with this genotype may to be more likely to have liver involvement, as most (i.e., 6/7) had abnormal findings on liver ultrasound. It is difficult, however, to draw any strong conclusions given the limited number of cases reported.

Congenital disorder of glycosylation $1 \mathrm{a}(\mathrm{CDG} 1 \mathrm{~A})$ is another life-threatening multisystemic disease caused by autosomal recessive variants in the PMM2 gene [8]. Patients with CDG1A present early in life with dysmorphic features and neurologic manifestations, in addition to HI and cystic kidney disease, and it is thought to be secondary to abnormal systemic glycosylation. The phenotype expressed in our case and the previously reported series of patients with HIPKD is distinct from that of CDG1A as it is restricted to the kidney, pancreatic B cells, and the liver. It is suspected that the promotor variant found in our patient, and those previously described, may have an organ- or tissue-specific effect [3]. Our patient displayed the same variant in the promoter region of the PMM2 gene and demonstrated abnormalities limited to her pancreas, liver, and kidneys. None of the patients previously described, or our own, followed the typical course of ARPKD or had mutations in the genes typically responsible for HI.

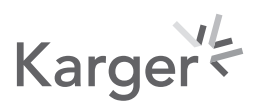




\section{Conclusion}

To our knowledge, this represents the first reported case in the USA of a patient with HIPKD due to variants in the PMM2 gene. We present this patient to alert neonatologists and pediatricians to the possibility of this rare disorder in newborns who present with either persistent hypoglycemia or polycystic kidneys detected pre- or postnatally. While there is a limited number of known cases of this disorder, it appears there may be long-term sequelae that require close follow-up from an early age.

\section{Statement of Ethics}

Case reports are educational in nature and are considered exempt by the Medical IRB of Marshal University. This exempt status does not require permission from the subject, or his or her legal guardian(s), for case reports unless HIPAA-protected identifying images or information are being shared. All medical images and information have been deidentified to protect the identity of our patient, and verbal permission was obtained from both parents prior to initiating this case report.

\section{Conflict of Interest Statement}

The authors have no conflicts of interest - personal, professional, or financial - to disclose.

\section{Funding Sources}

There were no internal or external funding sources for this report.

\section{Author Contributions}

Priya Prasher and Katherine Redwood are responsible for the design of this work, acquisition and analysis of data, draft and critical revisions, and final approval. They are accountable for this work in its entirety. Hillarey Stone and Edward Nehus are responsible for analysis of data, draft and critical revisions, and final approval. They are accountable for this work in its entirety. James R. Bailes is responsible for the design of this work, draft and critical revisions, and final approval. He is accountable for this work in its entirety. Deborah L. Preston is responsible for analysis of data, draft and critical revisions, and final approval. She is accountable for the work in its entirety. Joseph Werthammer is responsible for the design of this work, acquisition and analysis of data, draft and critical revisions, and final approval. He is accountable for the work in its entirety.

\section{References}

1 Palladino AA, Stanley CA. A specialized team approach to diagnosis and medical versus surgical treatment of infants with congenital hyperinsulinism. Semin Pediatr Surg. 2011 Feb;20(1):32-7.

2 Hartung EA, Guay-Woodford LM. Autosomal recessive polycystic kidney disease: a hepatorenal fibrocystic disorder with pleiotropic effects. Pediatrics. 2014 Sep;134(3):e833-45.

3 Cabezas OR, Flanagan SE, Stanescu H, García-Martínez E, Caswell R, Lango-Allen H, et al. Polycystic kidney disease with hyperinsulinemic hypoglycemia caused by a promoter mutation in phosphomannomutase 2 . J Am Soc Nephrol. 2017 Aug;28(8):2529-39. 
4 Guay-Woodford LM, Bissler JJ, Braun MC, Bockenhauer D, Cadnapaphornchai MA, Dell KM, et al. Consensus expert recommendations for the diagnosis and management of autosomal recessive polycystic kidney disease: report of an international conference. J Pediatr. 2014 Sep;165(3):611-7.

5 Shneider BL, Magid MS. Liver disease in autosomal recessive polycystic kidney disease. Pediatr Transplant. 2005 Oct; $9(5): 634-9$.

6 Flanagan SE, Kapoor RR, Hussain K. Genetics of congenital hyperinsulinemic hypoglycemia. Semin Pediatr Surg. 2011 Feb;20(1):13-7.

7 Mohamed Z, Arya VB, Hussain K. Hyperinsulinaemic hypoglycaemia:genetic mechanisms, diagnosis and management. J Clin Res Pediatr Endocrinol. 2012 Dec;4(4):169-81.

8 Grünewald S. The clinical spectrum of phosphomannomutase 2 deficiency (CDG-Ia). Biochim Biophys Acta. 2009 Sep;1792(9):827-34. 